\title{
Pressing information
}

\author{
One aim in science communication is to make the general public more aware of the breakthroughs and insights \\ basic science research provides. Our press office gives us one route to help achieve that goal.
}

A s we've expressed several times in past editorials, it is important for scientists to find a way to effectively communicate with the general public. This serves to emphasize the importance of funding basic science research and to increase public awareness of scientific findings and of how they relate to and may improve everyday life and the world around us. NSMB, as one of the Nature Research Journals and part of Nature Publishing Group (NPG), already has an effective system in place to notify the media, and thus the public, of the research published in our pages.

For those who may not have noticed, NSMB recently made a relatively big splash in US and international news outlets with the publication of an article from Sui, Donis, Liddington, Marasco and colleagues (http:// www.nature.com/nsmb/journal/v16/n3/abs/nsmb.1566.html) on the isolation of monoclonal antibodies that could recognize a variety of influenza strains, paving the way for a broad-spectrum therapy against the flu and giving hope for the development of a long-soughtafter universal flu vaccine. If you haven't seen some of the wonderful coverage the research received, don't worry, we've been keeping track of it all (http://www.nature.com/nsmb/news.html).

Although the credit for this research and breakthrough goes to the authors, who performed and analyzed the experiments, a lot of credit for getting the word out should definitely go to the NPG press office (http://press.nature.com), which sends press releases for articles published by all Nature-branded journals and helps coordinate press coverage by members of the science media, providing another service to our authors (http://www.nature.com/authors/author_benefits/ index.html). With press offices based in the US and UK, our press officers serve as first contacts for journalists both local and abroad, and are wardens of our press and embargo policies.

The NPG press office is notified of every manuscript that is accepted for publication and is responsible for coordinating the press release of manuscripts once publication dates have been set. For the Nature research journals, research articles are published online every Sunday, and the news embargo lifts at the time of publication on the journals' websites, at 1 p.m. US Eastern time (6 p.m. London time). The press office performs a variety of tasks as each article is prepared for publication. Importantly for our authors, the press office provides additional notification to the authors of a research paper the Tuesday before the particular Sunday a paper is scheduled to appear online, informing them of our embargo policies, which are strictly enforced.

In a recently added service, press officers also contact relevant funding agencies and home institutions involved with the work. This gives adequate time for internal coverage by institutional public information or public affairs offices. Advance notice is particularly useful for large agencies, such as the National Institutes of Health, that participate in a large number of research programs and may therefore be contacted about any number of forthcoming research publications.

With backgrounds in the biological and physical sciences, the NPG press officers write the press releases for newsworthy articles published in Nature. They also work with the editors at the Nature research journals to compose press releases for articles that may have wide public appeal. (Usually, articles whose findings have a direct connection to a disease may garner attention, though it can be difficult to predict what will catch the eyes of science journalists.) Author contact details accompany the highlighted papers within a release, along with a list of all papers that are being published in that journal in that particular week. On the Tuesday before the articles will be published online, the press office e-mails a compiled release covering Nature and the Nature research journals for that week to over 3,000 science journalists and media organizations. In some special cases, the press office will also organize a press briefing, at which journalists can speak directly to the researchers about their work.

The goal for all this work by our press offices is to make the science we publish accessible to the public by directly connecting the researchers with the journalists who report upon their work, and to do so in a way that does not distort, hype or dilute the scientific message. Although these are important steps toward getting science to the public, a certain limitation is present at the receiving end, given that the media covers only certain types of stories (see the recent Nature Methods editorial (http://www.nature.com/nmeth/journal/v6/ n3/abs/nmeth0309-181.html) for more details on this).

A bright spot aimed at opening up more science to journalists is the Science Media Centre (SMC, http://www.sciencemediacentre.org). The SMC is a nonprofit organization that has become a valuable resource for UK-based journalists looking to report on late-breaking science news stories, directing them to scientists who would be appropriate to comment on the story and providing them with the salient scientific points involved in the story, almost like our own press office but on a national scale. Perhaps with all the money being injected into US-based research in the immediate future - and to add another suggestion to the thousands of ideas out there for how this windfall should be spent and allocated-some money should be directed toward developing a US organization similar to the SMC, which would be perfectly in line with the current administration's goals of open communication and "restoring science to its rightful place." 